monitor of map the intersection(s) of nephrology data in relation to CKD via wearable technology (MI band and Yu band). To study effects of daily life routine activities on data by a wearable device that can obtain real-time CKD data, help technologists understand medical aspects, and clinicians to understand technological processes them and provide assistance based on pre-determined specifications in CKD patients in New Delhi, India.

Methods Total of $78 \mathrm{CKD}$ patients were taken as a subject with an equal ratio of male and female at university college of medical sciences, New Delhi, India. Wearable monitoring devices were put on the wrist of CKD patients for 30 days, and a questionnaire was filled out by each patient. Both diabetes and cardiovascular disease, in turn, are known as important factors for developing CKD and aggravation toward once end-stage renal disease. In all subjects, blood glucose was measured on daily basis with day to day data of their monitoring of step count, blood pressure, calorie burnt, insulin dose, motion time i.e. every time when your body was in motion, sleep monitoring (deep sleep, light sleep, wake up time), monitoring heart rate, cardiac arrhythmias to know daily routines and recording them for health purpose.

Results Present results showed that both wearable device readings showed there was a normal heart rate, more calorie burnt with better control of sugar control and average good sleep count in more physically workout, include walking in stroke patients compared to less physically workout CKD patients, identified by professional physiotherapists. Both device readings showed that after changing lifestyle routine among less physically active CKD patients, their post- CKD events normalise.

Conclusions With this study we show that, by using, these wearable devices ensured online assistive feedback for CKD patients is possible with their health awareness, exercising and motivate further studies.

\section{IDDF2018-ABS-0265 BENEFICIAL EFFECTS OF SILYBUM MARIANUM SEED EXTRACT AGAINST HEPATIC FIBROSIS INDUCED BY CARBON TETRACHLORIDE IN RATS}

Sanjay Kumar*, RK Khanna. Department of Basic and Applied Biology, Vivekananda Global University, Jaipur, Rajasthan, India

\subsection{6/gutjnl-2018-IDDFabstracts.56}

Background Silybum marianum or milk thistle is the most well-researched plant in the treatment of liver disease. Silymarin, derived from the milk thistle plant, silybum marianum, has been used for centuries as a natural remedy for diseases of the liver and biliary tract. The current study aimed to investigate the silybum marianum seed extract as hepatoprotective agent verse hepatic damages caused by carbon tetrachloride $\left(\mathrm{CCl}_{4}\right)$.

Methods Male Wistar albino rats were divided into two equal groups $(n=8)$ and treated as follows: Group 1, kept as control group and orally given saline; Group 2, kept as control positive and were administered daily oral doses of silymarin $(50 \mathrm{mg} / \mathrm{kg})$ daily for 21 days and subsequently injected i.p. with $\mathrm{CCl}_{4}(50 \% \mathrm{v} / \mathrm{v}$ in olive oil; $1 \mathrm{ml} / \mathrm{kg})$ on the $22 \mathrm{nd}$ day. $\mathrm{CCl}_{4}$-induced damages were assessed through liver function markers viz.; alkaline phosphatase (ALP), alanine transaminase (ALT), aspartate transaminase (AST), $\gamma$-glutamyltransferase $(\gamma$ -
GT) and lactate dehydrogenase (LDH). Changes in lipid profile were checked by measuring serum total cholesterol (TC), triglycerides (Tg), high-density lipoproteins (HDL) and lowdensity lipoproteins (LDL). Antioxidant status was checked by the activities of antioxidant enzymes (superoxide dismutase, glutathione peroxidase), DNA damages, malondialdehyde (MDA) and nitric oxide (NO) content. The histopathological changes were observed with Masson staining.

Results Administration $\mathrm{CCl}_{4}$ induced an elevation of serum amino- and glutamyl transferases activities and an increased peroxidation, as well as a decrease of superoxide dismutase and glutathione peroxidase activities in the liver. Administration of $\mathrm{CCl}_{4}$ in rats caused a significant increase in liver function and lipid profile indicating hepatic damages which were restored by co-administration of silymarin. Cellular and DNA damages in hepatic tissues were caused by $\mathrm{CCl}_{4}$ which shown clear hepatic fibrosis in addition to disturbing antioxidant enzyme level. Co-treatment with silymarin regulated these markers of oxidative dysfunctions. Silymarin enhances hepatic glutathione and may contribute to the antioxidant defence of the liver.

Conclusions It may be concluded that silymarin has the ability to reverse $\mathrm{CCl}_{4}$ - induced hepatic damages. Silymarin has been used to treat alcoholic liver disease, acute and chronic viral hepatitis and toxin-induced liver diseases.

\section{Clinical Gastroenterology}

\section{IDDF2018-ABS-0001 FOREIGN BODY INGESTION: 4 CASES WITH DIFFERENT MANAGEMENT APPROACH}

${ }^{1}$ Garima Gupta*, '2Deepak Kumar, ${ }^{2}$ Urmila Jhamb. ${ }^{1}$ Hindu Rao Hospital and NDMC Medical College, New Delhi, India; ${ }^{2}$ Maulana Azad Medical College, New Delhi, India

\subsection{6/gutjn|-2018-IDDFabstracts.57}

Background We managed four cases of ingested foreign bodies located in oesophagus and stomach differently. There are specific guidelines for the management of FB ingested in paediatrics age group depending upon type, size, symptoms and location in the digestive tract.

Methods Our first two cases were of ingested button battery which is blunt or non-sharp type contain corrosives, small in size $(<2 \mathrm{~cm}$ diameter) and were impacted in the oesophagus, both the patients presented to us late by day 4 and 5 . Both presented with symptoms of a cough, dysphagia, vomiting and respiratory distress later diagnosed tracheoesophageal fistula and needed surgical correction. Our third case was a 5 years old patient who had ingested a coin, which is blunt in type, was exactly $2 \mathrm{~cm}$ in diameter and was located in the stomach. As per guidelines he was followed serially for location by $\mathrm{X}$ ray and symptoms but was later removed endoscopically, as coin had not passed the stomach outlet by the 4th week and had a risk of mucosal injury, although the patient was asymptomatic. Our fourth case had ingested small blunt plastic toy part which was initially impacted in oesophagus before referred to us. FB was tried to be removed endoscopically from oesophagus as soon as diagnosed with X-ray but was pushed down to the stomach during the procedure. In this case, the patient was further observed by us since the FB reached the stomach which was later found passed spontaneously in stool on day 6 after endoscopic failure. 